\title{
An UCWW-enabled Mobile Geographic Information System
}

\author{
Ivan Ganchev, Máirtín O’Droma, and Sachin K. Agrawal
}

\begin{abstract}
This paper describes a Geographic Information System (GIS) for use in the Ubiquitous Consumer Wireless World (UCWW), whereby the consumers will be able to acquire geographic information adapted to the capabilities of their mobile terminals anytime-anywhere-anyhow. Third-party Authentication, Authorization, and Accounting service providers (3P-AAA-SPs) handle all authentication, authorization, accounting, charging and billing related issues between the consumers and GIS service providers. The UCWW-GIS architecture is proposed and explained. Sample signaling flows between the main entities involved in a typical UCWW-GIS service session are described.
\end{abstract}

Index Terms-ArcGIS tool, geographic information system (GIS), ubiquitous consumer wireless world (UCWW).

\section{INTRODUCTION}

This paper discusses the use of a mobile geographic information system (GIS) by the consumers to acquire geographic information anytime-anywhere-anyhow in the ubiquitous consumer wireless world (UCWW) [1], [2].

The UCWW is a consumer-centric generic techno-business model foundation for future generations of wireless communications. It is an evolution of, and alternative to, the legacy subscriber-based and network-centric model. Proposed by the Telecommunications Research Centre, University of Limerick, Ireland, the UCWW has two key aspects: 1) a person-centric IPv6 address enabling full number portability; and 2) an access-network-independent third-party authentication, authorization, and accounting (3P-AAA) service provision, which will empower the consumers to opt out of their long-term subscriptions with access network providers and use advertised communication services from any consumer-centric wireless access network that is present to them. The most characteristic attributes of UCWW are the increased consumer choice of services, consumer-driven always best connected and best served (ABC\&S), consumer-driven integrated heterogeneous networking (IHN), elimination of roaming charges, and a level "playing field" for new access network provider entrants [1], [2].

Mobile GIS is an integrated technological framework for accessing geographic data through mobile terminals (MTs), such as laptops, pocket PCs, personal digital assistants (PDAs), and smart phones [3]-[5]. Mobile GIS allows

This work was supported the Irish Research Council for Science, Engineering and Technology (IRCSET) and the Telecommunications Research Centre, University of Limerick, Ireland (http://www.ece.ul.ie/trc/).

I. Ganchev and M. O'Droma are with the Telecommunications Research Centre, University of Limerick, Ireland (e-mail: Ivan.Ganchev@ul.ie, Mairtin.ODroma@ul.ie). consumers to collect geographic information whenever they need it, visualize information in a digital map, and share it with the rest of the world, while also improving productivity and data accuracy [6].

This paper proposes an appropriate GIS architecture for use in the UCWW. It also describes the signaling flows between the main GIS entities for requesting and obtaining a map data generated by the GIS server up to the final charging and billing settlement.

\section{II.UCWW-GIS SYSTEM ARCHITECTURE}

Fig. 1 shows the proposed UCWW-GIS architecture consisting of the following components:

- Consumer's MT: This is used by the consumer to request and retrieve the desired geographic information, and interact directly with the GIS server [6]-[8] and the 3P-AAA-SP's server;

- 3P-AAA-SP's server: Once a request for a map is received by the GIS server, the 3P-AAA-SP's server assists the latter in completing the consumer's authentication, authorization and accounting, and subsequent charging and billing (C\&B). The provided 3P-AAA service is network-independent, autonomous, trusted, and pervasive [1], [2];

- GIS server: This publishes and integrates consumer-generated contents and geographic maps [7], [8]. The GIS server communicates from time to time with the 3P-AAA-SP's server for the authentication, authorization, accounting, charging and billing of the consumer.

\section{A. GIS Mobile Application}

The GIS mobile client uses a base map to display the surroundings and other geographic information. The client application has common GIS functionalities such as zoom-in, zoom-out, pan, identification etc. By using the map cache, consumers can retrieve information about point of interest and show this information on the mobile map [3]-[8].

An example of a GIS mobile client is the ArcPad Mobile GIS [4]. It supports various image formats such as Portable Network Graphics (PNG), Joint Photographic Experts Group (JPEG), Compressed ARC Digitized Raster Graphics (CADRG), MultiResolution Seamless Image Database (MrSID), and bit map (BMP) in a multilayered environment. The consumers can combine vector and raster data with the only limitations being the speed and memory capacity of the MT hardware [3]-[8]. ArcPad includes a comprehensive set of map navigation, search, query, and display tools including variable zoom and pan, fixed zoom, zoom to a specified layer and a spatial bookmark. These tools are designed to facilitate working with spatial data on MTs to receive and locate the desired locations on the received map [3]-[8]. 


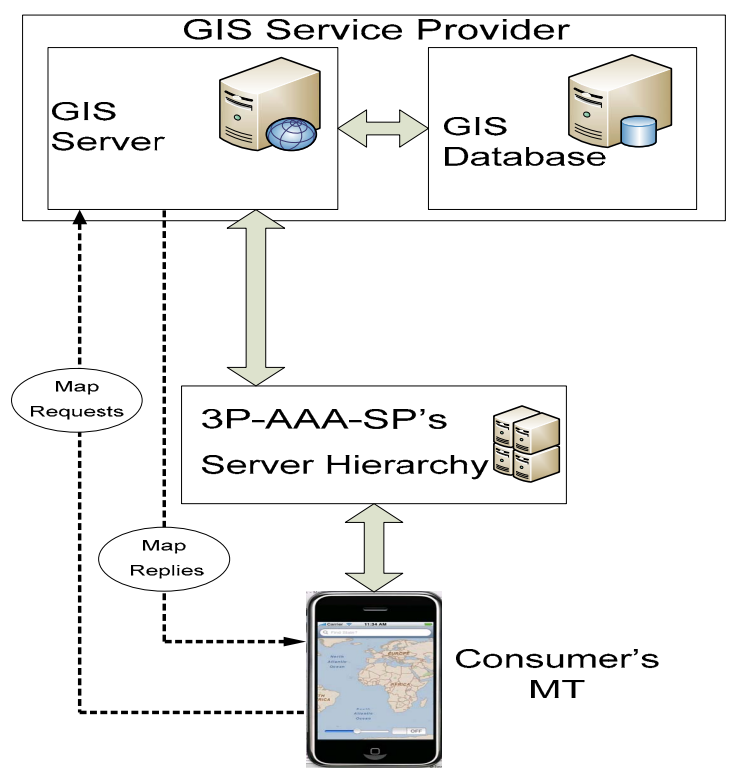

Fig. 1. The UCWW-GIS architecture.

A multi-application consumer identity module (CIM) card is inserted in the MT to support the 3P-AAA process. CIM has one or more 3P-AAA application clients installed. Simultaneous accounts with different 3P-AAA service providers (3P-AAA-SPs) are possible. The CIM card comes with generic 3P-AAA clients pre-installed and several free spaces on the card chip leasable to specific 3P-AAA-SPs for installing (and upgrading) their own specific 3P-AAA client, designated for that consumer [1], [2].

Through standardized protocols the 3P-AAA client on the MT interacts with the GIS server and the 3P-AAA-SP's server for mutual authentication and the exchange of security credentials. A part of these standardized protocols is the signaling to establish authentication securely prior to GIS service purchase, e.g. by employing the ITU-T's X.509 standard based on a public key infrastructure (PKI) [1], [2].

\section{B. 3P-AAA-SP's Server}

The 3P-AAA-SP's server incorporates also the C\&B aspects and acts as a certificate authority (CA) for issuing digital certificates for communicating parties [1], [2].

The 3P-AAA-SP's server can receive AAA requests from the consumer's MT or from the GIS server. If the server does not recognize the consumer, it will seek information from the next level on the hierarchy, and so on, eventually receiving back information to enable or reject (e.g., for insufficient credit) the continuation of the AAA procedure for the requesting consumer. Accounting information in relation to consumers (and usually confirmed by them) is collected from the GIS service provider and forwarded to the 3P-AAA-SP's server. At the end of the GIS service session, the 3P-AAA-SP's server generates a bill and charges the consumer accordingly as per his/her service usage.

\section{GIS Server}

The GIS server works in association with a GIS database. The GIS server controls the GIS database and performs operations like fetching/saving/updating of map data from/in the GIS database [3]-[8], [9]-[14].

The GIS server requests from the database geographic information (e.g. map data) according to the consumer's demand. If the requested map data is not available in the database, the GIS server generates the requested map data and delivers it to MT. The GIS server also updates the GIS database with the new map data for future use. The base maps are created by the GIS server, which prepares a mobile base map as a dataset that is optimized and ready for use with ArcGIS mobile applications [3]-[8].

The Create Mobile Base Map tool at the GIS server creates a folder of highly compressed map layers. The output from the tool consists of the following files (all files are deployed on MT, except where noted) [4], [7], [8], [12]:

- One or more Smart Data Compression (SDC) datasets $(<$ filename $>$.SDC $)$ containing the extracted vector data;

- A XML-based symbology file (<filename $>$.navmap) containing a reference to all extracted layers and the associated rendering characteristics (symbology, layer scale ranges, etc);

- An ArcMap document containing all non-extracted layers and layers' properties from the input map document. As the map document is not deployed on MT, it is written to the parent of the output folder. If all layers from the input map document are extracted, then no output map document will be created;

- (optional) A SDC-based Network Dataset rebuilt for the extracted area;

- (optional) One or more SDC-based Location Services and Address Locators rebuilt for the extracted area.

The highly compressed map layers are transmitted and copied to a storage card or onto the main memory of MT. The base map layers are static and rarely change. By creating a base map dataset, the MT optimizes the local storage of non-editable GIS data [4], [7], [8], [12].

The base map layers in MT provide reference information used for visualization and/or navigation purposes (aerial imagery, land parcel boundaries, streets, etc). There is no need to synchronize base map layers, so they can be provisioned onto MT independently of a specific field project [3]-[14].

\section{UCWW-GIS SIGNALING FLOWS}

Typical signaling flows between MT, GIS server, GIS database, and 3P-AAA-SP's server that occur during the UCWW-GIS service session are shown in Fig. 2. 


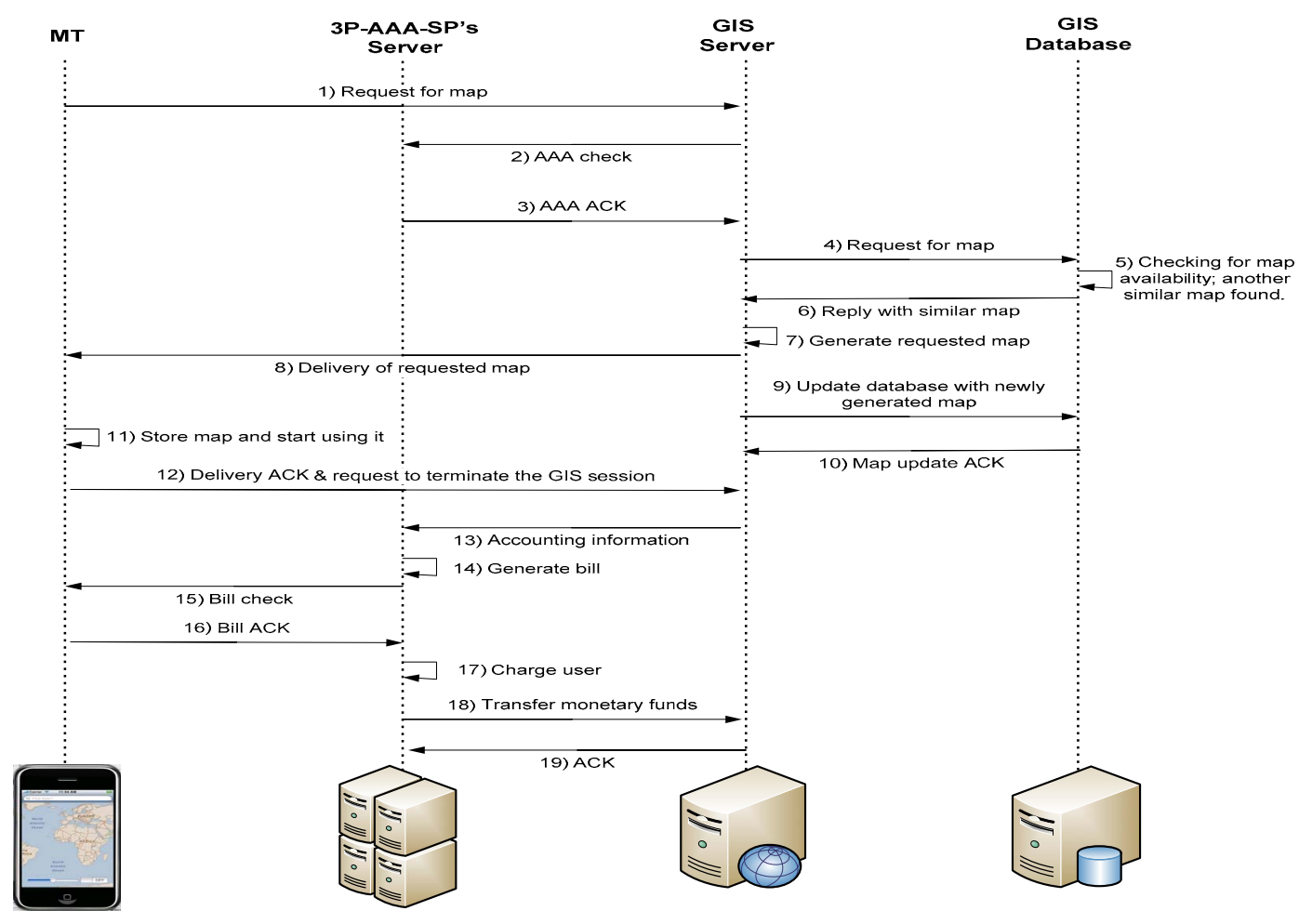

Fig. 2. Typical core UCWW-GIS signaling.

In our example, the consumer requests a map of the towns in the Limerick County (Ireland) along with the main roads between them:

1) MT transmits the consumer's request for map data to the GIS server.

2) The GIS server requests the initiation of the authentication, authorization, and accounting procedure from the 3P-AAA-SP's server.

3) The 3P-AAA-SP's server checks the consumer's credentials and, if these are found satisfactory, sends a positive acknowledgement (ACK) back to the GIS server.

4) The GIS server forwards the consumer's request to the GIS database.

5-6) After performing an internal search, the GIS database finds out that the requested map is not available. However, another similar map containing only the town names is found. So this is sent back to the GIS server.

7) The GIS server generates a new map based on the map received from the database. It also manipulates data according to the MT capabilities and creates a vector base map optimized for use with the ArcGIS mobile application.
8) The GIS server delivers the requested map data to MT. 9-10) The GIS server updates the GIS database with the newly generated map for future use and the database acknowledges this.

11-12) MT stores the map in its memory and sends back an ACK for the successful receiving of the map data along with a request to terminate the GIS service session. The ArcGIS mobile application retrieves the map data from the memory and displays it on the MT screen as shown in Fig. 3 with the town names and the roads.

13) The GIS server terminates the session with the MT and sends corresponding accounting information to the 3P-AAA-SP's server.

14-15) The 3P-AAA-SP's server generates the bill and sends it for checking to MT.

16) If the bill is found correct, MT acknowledges it.

17-18) The 3P-AAA-SP's server charges the consumer accordingly and transfers the relevant monetary funds from his/her account to the GIS server as a final settlement.

19) The GIS server confirms the successful completion of the charging and billing procedure. 


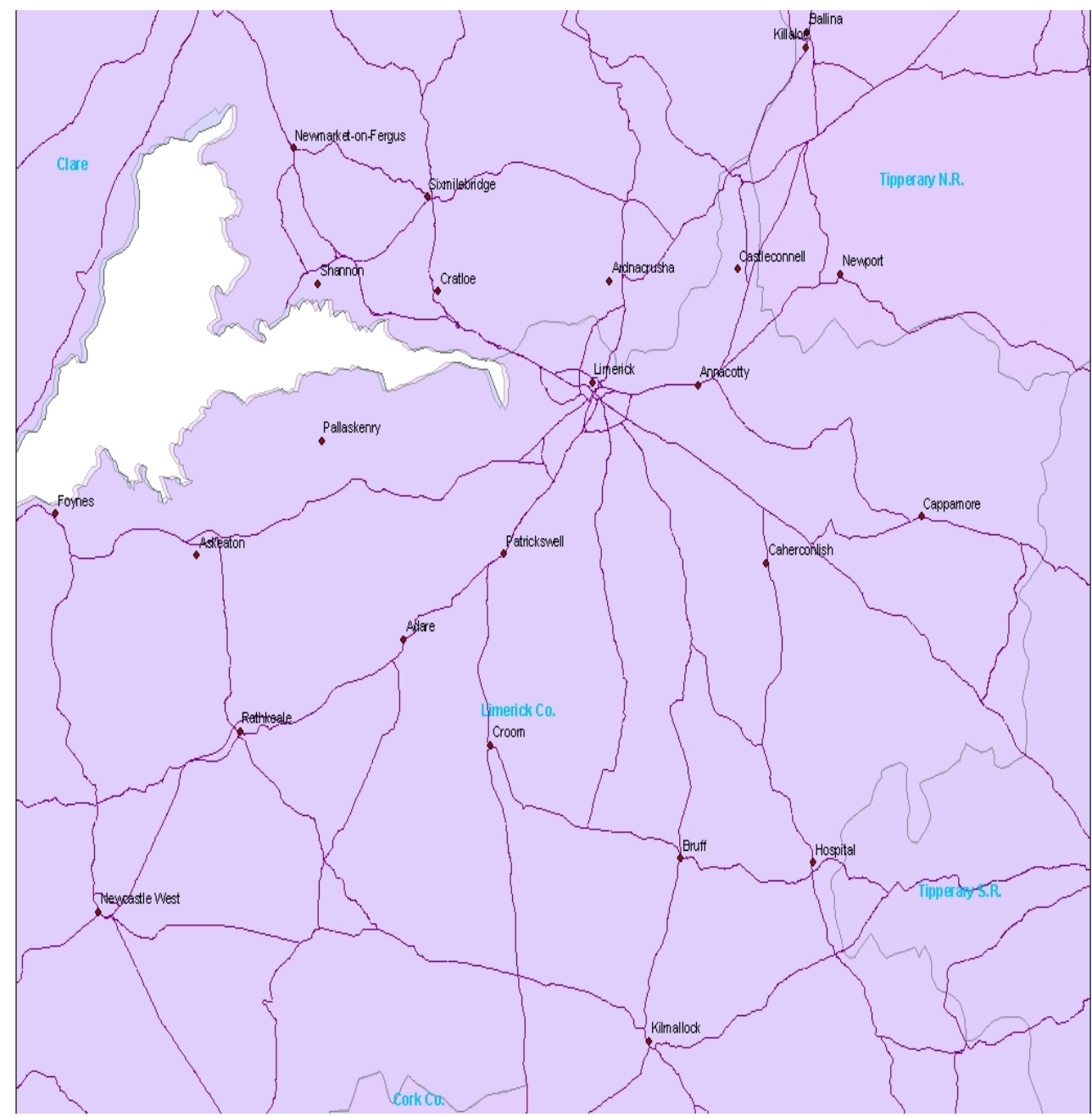

Fig. 3. A sample map requested by a consumer.

\section{CONCLUSION}

This paper has described a Geographic Information System (GIS) for use in the Ubiquitous Consumer Wireless World (UCWW). The UCWW-GIS architecture has been proposed and explained along with the core signaling flows between the main entities involved in a typical service session. Authenticated and authorized by a third-party authentication, authorization, and accounting service providers (3P-AAA-SPs), the consumers are able to acquire geographic information adapted to the capabilities of their mobile terminals anytime-anywhere-anyhow.

\section{REFERENCES}

[1] M. O'Droma and I. Ganchev, "The creation of a ubiquitous consumer wireless world through strategic ITU-T standardization," IEEE Communications Magazine, vol. 48, no. 10, pp. 158-165, 2010.

[2] M. O'Droma and I. Ganchev, "Toward a ubiquitous consumer wireless world," IEEE Wireless Communications, vol. 14, no. 1, pp. 52-63, 2007.

[3] S. Weidong and S. Guibo, "Using mobile GIS as volunteered GI provider," in Proc. 1st IEEE Conf. on Information Science and Engineering, pp. 2229-2232, 2009.

[4] “ArcPad®: mobile GIS," An ESRI White Paper, pp. 1-17. 2004 Available: http://www.esri.com/library/whitepapers/pdfs/arcpad.pdf

[5] M. H. Tsou, "Integrated mobile GIS and wireless internet map servers for environmental monitoring and management," Cartography and Geographic Information Science, vol. 31, no. 3, pp. 153-165, 2004.
[6] L. He, J. Wu, and X. Fu, "Design and implementation of GPS \& GIS based mobile navigation system," in Proc. IEEE Int. Conf. on Network Infrastructure and Digital Content, pp. 953-957, 2009.

[7] "ArcGIS server best practices and guidelines," NEARC2007 ESRI Technical Session, ESRI, Boston. Available: http://www.northeastarc.org/archive/2007/esritalks/Nearc07ArcGISSe rverBestPracticesGuidelines.pdf

[8] "ArcGIS desktop GIS," Available: http://www.esri.com/products/index.html\#desktop_gis_panel

[9] M. Zhang and J. Mei, "Design and implementation for mobile-based GIS urban information system," in Proc. IEEE Symp. On Information Science and Engineering, pp. 464-467, 2008.

[10] C. A. Davis, Y. J. Kim, and F. L. P. Duarte-Figueiredo, "OGC web map service implementation challenges for mobile computers," in Proc. 17th IEEE Int. Conf. on Geoinformatics, pp. 1-6, 2009.

[11] H. Li, F. Jin-hong and F. Xiao-ling, "Research and implementation of mobile navigation system based on GPS and GIS," in Proc. 1st IEEE Conf. on Information Science and Engineering, pp. 2129-2132, 2009.

[12] A. S. A. El-Swaify and D. P. Dunham, "Using ArcView to facilitate navy capital improvement planning in mainland Japan," in Proc. of ESRI, Available:

http://proceedings.esri.com/library/userconf/proc00/professional/pape rs/pap188/p188.htm

[13] J. Almeida, H. Fernandes, J. Barroso, and V. Filipe, "Web platform architecture to support the geographic information system of the University of Trás-os-Montes and Alto Douro Campus," in Proc. IEEE Conf. on New Trends in Information and Service Science, pp. 1112-1117. 2009.

[14] Y. Gang, J. Zhen-hong, Q. Xi-Zhong, C. Chun, L. Tao, W. Hao, and Z Jun-kai, "Research on positioning technology of Xinjiang mobile GIS," in Proc. IEEE Conf. on Environmental Science and Information Application Technology, pp. 441-444, 2009. 